\title{
Avaliação de depressão, problemas de comportamento e competência social em crianças obesas ${ }^{1}$
}

\author{
Evaluation of depression, behavioral problems \\ and social skills in obese children
}

\author{
Andreia Mara Angelo Gonçalves LUIZ² \\ Ricardo GORAYEB ${ }^{3}$ \\ Raphael Del Roio LIBERATORE JÚNIOR ${ }^{4}$
}

\begin{abstract}
Resumo
O objetivo deste estudo foi avaliar a ocorrência de depressão, problemas comportamentais e competência social em crianças obesas, comparativamente a crianças não obesas. Participaram do estudo 90 crianças, das quais 60 eram obesas e 30 não eram, com idade entre 7 e 13 anos. Na coleta de dados foram utilizados: Ficha de Identificação, Inventário de Depressão para Crianças e Inventário de Comportamentos da Infância e Adolescência. Os dados foram analisados quantitativamente, com testes estatísticos não paramétricos e paramétricos, com nível de significância de 0,05. Os resultados apontaram diferença significativa entre as crianças obesas e as crianças não obesas em relação às variáveis psicológicas estudadas. Na análise multivariada, a depressão e os distúrbios internalizantes foram as variáveis que mais discriminaram as crianças obesas das não obesas. Os dados indicam, portanto, a necessidade de uma intervenção precoce e a realização de programas de prevenção.
\end{abstract}

Unitermos: Depressão. Obesidade. Problemas de comportamento.

\begin{abstract}
The objective of the study was to evaluate the occurrence of depression, behavioral problems and social skills in obese children, contrasting to children who are not obese. Ninety children, ages between 7 and 13, participated in this study; 60 were obese and 30 were not. Data was collected using the following methods: Identity Cards; Children's Depression Inventory and the Child Behavior Checklist. Data analysis was performed quantitatively, using parametric and non-parametric statistical tests, with a significance level of 0.05. Results demonstrated a significant difference between obese and non-obese children, as regards the psychological variables studied. Based on a multivante analysis, depression and internal disorders distinguished obese children from those who were not obese. The data indicates, however, that there is a need for early intervention and a need to establish programs of prevention.
\end{abstract}

Uniterms: Depression. Childhood obesity. Behavioral problems.

\section{urvt}

- Artigo elaborado a partir da dissertação de A. M. A. G. LUIZ, intitulada "Ansiedade, depressão, problemas de comportamento e competência social em crianças obesas". Universidade de São Paulo, 2004.

2 Faculdade de Medicina de São José do Rio Preto, Curso de Aprimoramento em Psicologia da Saúde, Hospital de Base, Serviço de Psicologia. Av. Brig. Faria Lima, 5416, VI São Pedro, 15090-000, São José do Rio Preto, SP, Brasil. Correspondência para/Correspondence to: A.M.A.G. LUIZ. E-mail: $<$ andreiamagl@hotmail.com>.

3 Universidade de São Paulo, Faculdade de Medicina de Ribeirão Preto, Departamento de Neurociências e Ciências do Comportamento. Ribeirão Preto, SP, Brasil.

4 Faculdade de Medicina de São José do Rio Preto, Serviço de Endocrinologia Pediátrica. São José do Rio Preto, SP, Brasil. 
A obesidade é um dos problemas de maior prevalência entre crianças e está aumentando de forma significativa em todo o mundo. É, por isso, considerada um grave problema de saúde pública (Mello, Luft \& Meyer, 2004).

Nos países desenvolvidos, a obesidade infantil atinge proporções epidêmicas, começando a substituir a desnutrição e as doenças infecciosas, o que a torna um fator significativo como problema de saúde. Esses países têm concentrado seus esforços na área de saúde pública e na prevenção das doenças não transmissíveis, dando ênfase à redução da obesidade pela modificação do padrão alimentar e redução do sedentarismo (Brownell \& O'Neil, 1999).

No Brasil, um estudo realizado no Rio Grande do Sul revelou que a prevalência de sobrepeso e obesidade em escolares de 11 a 13 anos foi elevada (24,8\%) (Suné, Dias-da-Costa, Olinto \& Pattussi, 2007). Outro estudo com adolescentes apontou maior prevalência de obesidade no sexo feminino com nível socioeconômico mais alto e de regiões industrializadas (Neutzling, Taddei, Rodrigues \& Sigulen, 2000).

É importante lembrar que a obesidade infantil é um problema tanto da área da saúde como cultural, pois mudanças sociais têm levado a uma alimentação rápida e inadequada, colaborando para o aumento do sobrepeso e obesidade, afetando, assim, a qualidade de vida desses indivíduos (Strauss, 1999).

A ocorrência de problemas psicossociais e transtornos psiquiátricos, juntamente com a obesidade infantil, bem como o impacto destes sobre o funcionamento da criança, indica a relevância de se estudar esse tema. Entre estes transtornos psiquiátricos, a depressão tem sido muito estudada em crianças e adolescentes obesos, e pode interferir em vários aspectos da vida da criança, prejudicando o rendimento escolar e o relacionamento familiar e social (Erermis et al., 2004; Lee, Cutatolo \& Freedsich, 2000; Luiz, Gorayeb, Liberatore Júnior \& Domingos, 2005; van Vlierberghe, Braet \& Mels 2008).

Csabi, Tenyi e Molnar (2000) compararam a presença de sintomas depressivos entre crianças obesas em tratamento ambulatorial e crianças não obesas; os resultados mostraram uma maior proporção de sintomas depressivos nas crianças obesas. Em outro estudo 42 com crianças entre 11 e 13 anos foi investigada a relação entre Índice de Massa Corporal (IMC), sedentarismo e sintomas depressivos, e os resultados apontaram uma correlação entre alto nível de sedentarismo, IMC com percentil elevado e sintomas de depressão (Anton et al., 2006).

Alguns estudos também sugerem que problemas relacionados às áreas sociais e comportamentais são mais comuns em crianças obesas. Os obesos sofrem maior discriminação e estigmatização social, interferindo no seu funcionamento físico e psíquico, o que pode causar um impacto negativo em sua qualidade de vida (Khaodhiar, McCowen \& Blackburn, 1999).

Ao longo da vida o excesso de peso traz outras dificuldades, como a diminuição da oferta de empregos, timidez e problemas de relacionamento afetivo. Devido a tais dificuldades, os indivíduos obesos sofrem ou impõem-se restrições diante de atividades rotineiras, como ir à escola, fazer determinados exercícios físicos, procurar emprego, comprar roupas, namorar e divertirse (Damiani, 2002). Podem também apresentar baixa autoestima, o que afeta o desempenho escolar e social (Abrantes, Lamounier \& Colosimo, 2002; Keller \& Steves, 1996).

Stradmeijer, Bosch, Koops e Seidell (2000), estudando a relação entre funcionamento familiar e problemas comportamentais na obesidade infantil, verificaram que tanto os pais como os professores apontaram maiores problemas comportamentais nos obesos. Outro estudo mostrou que a maioria das crianças obesas apresentava problemas sociais, quando avaliadas por meio de questionário respondido pelos pais após solicitação do pesquisador (Epstein, Meyers \& Anderson, 1996).

Analisando a literatura, percebe-se que é encontrada uma forte relação entre a obesidade e aspectos psicológicos, tais como sintomas depressivos, problemas comportamentais e sociais, independentemente da sua conotação de causa ou consequência envolvida no processo da obesidade.

Considerando que a depressão, problemas comportamentais e competência social são, com frequência, associados à obesidade, este estudo teve como objetivos:a) avaliar a presença de sintomas depressivos, competência social e distúrbios comportamentais em crianças com obesidade, comparativamente a crianças não obesas; b) caracterizar o perfil dos participantes. 


\section{Método}

\section{Participantes}

A amostra consistiu de 90 crianças, divididas em grupo de obesos e grupo-controle. O Grupo de Obesos (GO) foi composto por 60 crianças com obesidade exógena, com IMC maior que o percentil 95 (Centers for Disease Control and Prevention - CDC, 2007), sendo 33 meninos e 27 meninas, com idade entre 7 e 13 anos (Média-M=9,8; Desvio-Padrão- DP=1,73) e escolaridade de $1^{\text {a }}$ a $7^{\text {a }}$ série $(M=3,7 ; D P=1,74)$, atendidas no ambulatório de obesidade de um hospital-escola do interior do Estado de São Paulo; quanto à escolaridade dos pais, predominou entre o primário e o $1^{\circ}$ grau completo.

O Grupo Controle (GC) incluiu 30 crianças não obesas, apresentando IMC entre o percentil 25 e 75 (CDC, 2007), sem diagnóstico anterior de obesidade, sendo 17 meninos e 13 meninas, com idade entre 7 e 13 anos $(M=10,13 ; D P=1,89)$ e escolaridade de 1 a à 7 a série $(M=3,7$; $\mathrm{DP}=1,77)$, atendidas no ambulatório de otorrinolaringologia do mesmo hospital, com diagnóstico de doenças consideradas comuns nessa área (amidalite, faringite e rinite alérgica). A escolaridade dos pais predominante também foi entre o primário e o $1^{\circ} \mathrm{grau}$ completo.

Foram critérios de exclusão do estudo, crianças com deficiência intelectual ou transtornos psiquiátricos, tais como: esquizofrenia, transtornos alimentares e transtornos invasivos do desenvolvimento, bem como crianças em tratamento psicoterápico.

\section{Instrumentos}

1) Ficha de Identificação

2) Inventário de Depressão Infantil (CDI): é um instrumento de autorrelato que avalia sinais cognitivos, afetivos e comportamentais de depressão em crianças (Kovacs, 1982). Foi adaptado e normatizado para o contexto brasileiro por Gouveia (1995), que padronizou o instrumento com um ponto de corte de 18. Crianças com pontuação igual ou acima desse valor são consideradas com sintomas depressivos. O CDI revelou-se um instrumento unidimensional, com consistência interna e confiabilidade. Cada um de seus 20 itens tem três opções de respostas, e a criança deve assinalar aquela que melhor descreve como tem se sentido emocionalmente nas últimas duas semanas.

3) Inventário de Comportamentos da Infância e Adolescência (CBCL): é um instrumento que avalia o funcionamento psicossocial de crianças e adolescentes de 4 a 18 anos, respondido pelos pais ou responsáveis. Contém 138 itens: 20 que avaliam competência social e 118 que avaliam problemas de comportamento. A primeira parte do questionário refere-se à competência social e investiga a participação da criança em atividades (esportes, brincadeiras, jogos, passatempos, trabalhos e tarefas), em organizações (clubes e times), seu relacionamento (família e amigos), independência para brincar e realizar tarefas de trabalho e desempenho na escola. Todas essas áreas juntas são denominadas Competência Social Global. A segunda parte abrange problemas emocionais e comportamentais, divididos em distúrbios internalizantes e externalizantes. Os internalizantes incluem ansiedade, depressão, retraimento, queixas esquizoides e somáticas. Os externalizantes são comportamentos delinquentes, cruéis ou agressivos. Os escores obtidos indicam o perfil social e comportamental da criança, e escores de corte determinam sua inclusão nas categorias não clínica (inferiores a 60), limítrofe (60 a 63) e clínica (superiores a 63). A inclusão da categoria limítrofe na categoria clínica reduz as categorias para apenas duas: não clínica e clínica, como foi adotado neste estudo (Bordin, Mari \& Caieiro, 1995).

\section{Procedimentos}

O estudo foi executado dentro das normas de ética em pesquisa em seres humanos. Os participantes foram abordados durante os dias de funcionamento dos ambulatórios. Após a autorização, o pesquisador aplicava os instrumentos individualmente. Coletaram-se os dados durante o período de um ano.

Os dados foram analisados quantitativamente, com procedimentos estatísticos não paramétricos e paramétricos, por meio dos programas estatísticos Statistical Package for the Social Sciences (SPSS), versão 10.0, e System for Statistics (SYSTAT), versão 7.0.

Para a análise univariada, na qual se verificou como a variável grupo (obesos e controle) se relacionava com as demais variáveis isoladamente, foi utilizado o 
Teste do Qui-Quadrado e o Teste Exato de Fisher. O nível de significância adotado foi de 0,05. Efetuou-se uma análise multivariada com o objetivo de observar como algumas variáveis interagiam com a variável grupo (obesos e controle), levando em consideração as inter-relações existentes. Foi empregado um método denominado Análise Discriminante, que visou, dentre as variáveis inicialmente postadas na análise, selecionar as que mais contribuíram (ou a combinação delas) para a diferenciação entre o grupo de obesos e o grupo controle. Para essa análise, foi utilizado o valor próprio dos instrumentos empregados no estudo.

\section{Resultados}

A Tabela 1 apresenta o perfil dos participantes do estudo. A análise comparativa entre os grupos mostrou que não houve diferença significativa em relação ao sexo, à idade e escolaridade das crianças e escolaridade dos pais. As mães das crianças obesas apresentaram porcentagem significativamente superior de escolaridade, entre o $2^{\circ}$ grau completo e o $3^{\circ} \mathrm{grau}$ completo, quando comparadas com as mães das crianças não obesas.

\section{Avaliação do Inventário de Depressão Infantil}

Os resultados do CDI (Tabela 2) indicam que os participantes do GO apresentaram 43,3\% de respostas com escore igual ou acima do ponto de corte para depressão, e os participantes do GC apresentaram 3,3\% de respostas com escores acima do ponto de corte. Esta diferença é estatisticamente significativa.

Tabela 1. Perfil dos participantes. São José do Rio Preto (SP), 2004.

\begin{tabular}{|c|c|c|c|c|c|c|}
\hline & \multirow[t]{2}{*}{ Variáveis } & \multirow[t]{2}{*}{ Especificações } & \multicolumn{2}{|c|}{$\begin{array}{c}\text { Grupo de } \\
\text { obesos }\end{array}$} & \multicolumn{2}{|c|}{$\begin{array}{c}\text { Grupo de } \\
\text { obesos }\end{array}$} \\
\hline & & & $\mathrm{n}$ & $\%$ & $\mathrm{n}$ & $\%$ \\
\hline \multirow{6}{*}{ 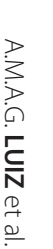 } & \multirow[t]{2}{*}{ Sexo } & Masculino & 33 & 55,0 & 17 & 56,7 \\
\hline & & Feminino & 27 & 45,0 & 13 & 43,3 \\
\hline & \multirow[t]{2}{*}{ Idade } & $7-10$ anos & 35 & 58,3 & 17 & 56,7 \\
\hline & & $11-13$ anos & 25 & 41,7 & 13 & 43,3 \\
\hline & \multirow[t]{2}{*}{ Escolaridade } & $1^{\mathrm{a}}$ - $4^{\mathrm{a}}$ série & 34 & 56,7 & 18 & 60,0 \\
\hline & & $5^{a}-7^{a}$ série & 26 & 43,3 & 12 & 40,0 \\
\hline \multirow{2}{*}{ 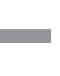 } & \multirow{2}{*}{$\begin{array}{l}\text { Escolaridade } \\
\text { da mãe }\end{array}$} & Primário - $1^{\circ} \mathrm{grau}$ & 37 & 61,7 & 28 & 96,3 \\
\hline & & $2^{\circ} \mathrm{grau}-3^{\circ} \mathrm{grau}$ & 23 & $38,3^{*}$ & 2 & 6,7 \\
\hline
\end{tabular}

$p<0,001 ;{ }^{*}$ Diferença estatisticamente significativa.
Em relação à Avaliação do Inventário de Comportamentos da Infância e Adolescência (CBCL), a Tabela 3 mostra os resultados no que se refere à Competência Social Global (CS-global), conforme a classificação "clínica" e "não clínica", proposta pelo autor do instrumento.

As crianças obesas apresentaram percentual estatisticamente maior de CS-global, classificada como "clínica" (63,3\%), quando comparadas com as crianças não obesas.

Os distúrbios comportamentais foram avaliados como distúrbios internalizantes, externalizantes e totais. A Tabela 4 apresenta os resultados dos Distúrbios Internalizantes (DI) e dos Distúrbios Externalizantes (DE) dos participantes.

Tabela 2. Distribuição das pontuações indicativas de sintomas depressivos nos participantes do GO e GC. São José do Rio Preto (SP), 2004

\begin{tabular}{lcccccc}
\hline \multirow{2}{*}{ Posição em relação ao ponto de corte } & \multicolumn{3}{c}{ GO } & & \multicolumn{2}{c}{ GC } \\
\cline { 2 - 3 } \cline { 6 - 7 } & & F & $\%$ & & $F$ & $\%$ \\
\hline Abaixo (sem depressão) & 34 & 56,7 & & 29 & 96,7 \\
Igual ou acima (com depressão) & & 26 & $43,3^{*}$ & & 1 & 3,3 \\
\hline
\end{tabular}

$p<0,001$; "Diferença estatisticamente significativa.

F: frequência; GO: grupo de obesos; GC: grupo-controle.

Tabela 3. Distribuição dos participantes em relação à CS-global. São José do Rio Preto (SP), 2004

\begin{tabular}{lcccccc}
\hline & \multicolumn{3}{c}{ GO } & & \multicolumn{2}{c}{ GC } \\
\cline { 2 - 3 } \cline { 5 - 6 } \cline { 5 - 6 } & $F$ & $\%$ & & $F$ & $\%$ \\
\hline Não clínica & 22 & 36,7 & & 27 & 90,0 \\
Clínica & 38 & $63,3^{*}$ & & 3 & 10,0 \\
\hline
\end{tabular}

$p<0,001$ *Diferença estatisticamente significativa.

F: frequência; GO: grupo de obesos; GC: grupo-controle; CS-global: competência social global.

Tabela 4. Classificação do GO e do GC como "clínica" e "não clínica" em relação aos DI e DE. São José do Rio Preto (SP), 2004.

\begin{tabular}{|c|c|c|c|c|c|c|c|c|}
\hline & \multicolumn{4}{|c|}{$\mathrm{DI}$} & \multicolumn{4}{|c|}{$\mathrm{DE}$} \\
\hline & \multicolumn{2}{|c|}{$\mathrm{GO}$} & \multicolumn{2}{|c|}{ GC } & \multicolumn{2}{|c|}{$\mathrm{GO}$} & \multicolumn{2}{|c|}{ GC } \\
\hline & $\mathrm{F}$ & $\%$ & $\mathrm{~F}$ & $\%$ & $\mathrm{~F}$ & $\%$ & $\mathrm{~F}$ & $\%$ \\
\hline Não clínica & 22 & 36,7 & 3 & 100,0 & 47 & 78,3 & 30 & 100,0 \\
\hline Clínica & 38 & $63,7^{*}$ & 0 & 0,0 & 13 & $21,7^{*}$ & 0 & 0,0 \\
\hline
\end{tabular}

$p<0,001$; "Diferença estatisticamente significativa.

F: frequência; GO: grupo de obesos; GC: grupo-controle; DI: distúrbios internalizantes; DE: distúrbios externalizantes. 
As crianças obesas apresentaram um número considerado estatisticamente maior de classificação "Clínica" (63,7\%) no que se refere aos DI em relação às crianças do GC. Quanto aos distúrbios externalizantes, no GO, 21,7\% das crianças foram classificadas como "clínicas", e nenhuma criança do GC foi classificada como "clínica". Portanto, as crianças do GO apresentaram porcentagem estatisticamente maior em relação à presença de distúrbios externalizantes, na comparação entre os grupos.

A Tabela 5 apresenta a classificação dos Distúrbios Comportamentais Totais (DT) dos participantes do GO e GC. Entre as crianças obesas, 60,0\% apresentaram DT classificados como "clínicos"; já no grupo controle nenhuma criança foi classificada como "clínica". Na comparação entre o GO e o GC, as crianças obesas apresentaram porcentagem estatisticamente superior de DT classificado como "clínico".

A análise multivariada, também realizada, permitiu identificar que as variáveis depressão e distúrbios comportamentais internalizantes encontravam-se presentes concomitantemente como descritoras do grupo de crianças obesas.

\section{Discussão}

Os dois grupos avaliados (grupo de obesos e grupo controle) apresentaram características semeIhantes em relação aos aspectos demográficos, o que trouxe maior consistência aos dados e permitiu fazer comparações entre os grupos. Assim, a variável obesidade permanece como a característica diferente entre os dois grupos.

Evidenciou-se, na avaliação do CDI, maior número de crianças obesas com sintomas de depressão,

Tabela 5. Classificação das respostas dos participantes do GO e do GC como "clínicas" e "não clínicas", em relação aos DT. São José do Rio Preto (SP), 2004

\begin{tabular}{lcccccc}
\hline & \multicolumn{3}{c}{ GO } & & \multicolumn{2}{c}{$G C$} \\
\cline { 2 - 3 } \cline { 5 - 6 } & $F$ & $\%$ & & $F$ & $\%$ \\
\hline Não clínica & 24 & 40,0 & & 30 & 100,0 \\
Clínica & 36 & $60,0^{*}$ & & 0 & 0,0 \\
\hline
\end{tabular}

$p<0,004{ }^{*}$ Diferença estatisticamente significativa.

F: frequência; GO: grupo de obesos; GC: grupo-controle; DT: distúrbio totais. quando comparadas com as crianças não obesas. A literatura também aponta que a depressão pode ser encontrada entre as crianças obesas, assim como nas portadoras de outros problemas crônicos de saúde, distúrbios endocrinológicos e neurológicos (Anton et al., 2006; Miyazaki, 1993; Rose, 1988; van Vlierberghe et al., 2008).

Erickson, Robinson, Haydel e Killen (2000) realizaram um estudo com pré-adolescentes obesos para investigar a relação entre depressão e obesidade e concluíram haver maior presença de depressão entre os adolescentes obesos do sexo feminino. Mustillo et al. (2003) estudaram, em crianças entre 9 e 16 anos, a associação entre obesidade e transtornos psiquiátricos. Os dados desse estudo mostraram que tanto as crianças do sexo feminino como masculino apresentavam obesidade associada com transtorno desafiador opositivo, mas os transtornos depressivos foram encontrados apenas entre os meninos.

Observa-se, então, uma maior proporção de sintomas depressivos entre as crianças obesas do que entre as de peso adequado e algumas diferenças entre os sexos, não havendo, portanto, um consenso, já que alguns estudos apontam uma permanência de sintomas depressivos entre as meninas, e outros, entre os meninos. Possivelmente, alguns estudos mencionam maior presença de sintomas depressivos entre as mulheres pelo fato de os transtornos alimentares, como a bulimia e a anorexia, serem mais predominantes no sexo feminino, devido à preocupação excessiva com a imagem corporal (Candy \& Fee, 1998; Erickson et al., 2000).

Em relação aos resultados do $C B C L$ o mesmo ocorreu: houve uma considerável associação entre déficits de competência social de forma global e obesidade infantil. Esse dado é compatível com a literatura sobre funcionamento social e doenças crônicas na infância, que evidencia que as crianças que sofrem de alguma doença crônica estão mais suscetíveis a apresentar problemas sociais, como dificuldade de relacionamento com amigos e familiares (Miyazaki, 1993). Além disso, os indivíduos obesos podem sofrer maior discriminação e estigmatização social. As crianças obesas, por exemplo, são comumente importunadas pelos colegas e menos aceitas socialmente do que as crianças com peso adequado. Tais dificuldades enfrentadas pelos obesos levam ao sofrimento e a restrições 
diante de atividades rotineiras, como ir à escola, fazer determinados exercícios físicos, procurar emprego, comprar roupas, namorar e divertir-se (Damiani, 2002; Ferriani, Dias, Silva \& Martins, 2005; Khaodhiar et al., 1999).

Faz-se necessário salientar que a competência social é uma habilidade básica para o funcionamento adequado na vida adulta e que a criança é considerada socialmente competente quando seu comportamento é avaliado como adaptativo à sua faixa etária. Esta habilidade é necessária para a aquisição de reforçamentos sociais, uma vez que crianças que não possuem comportamentos sociais apropriados são, com frequência, isoladas e rejeitadas, e podem apresentar problemas emocionais (Falcone, 2000).

Os distúrbios comportamentais, que compreendem tanto os distúrbios internalizantes como os externalizantes, assim como os distúrbios totais, também demonstraram diferença significativa nas crianças estudadas.

Deste modo, além da percepção da criança, avaliada pelo CDI, as mães das crianças obesas também perceberam seus filhos com comprometimento em relação aos distúrbios internalizantes. Este dado é compatível com outros estudos, que indicam que as crianças e adolescentes obesos, quando avaliados por seus pais ou pelo $C B C L$, evidenciam presença significativa de sintomas internalizantes (Erermis et al., 2004; van Vlierberghe et al., 2008).

Esses dados revelam que as crianças obesas avaliadas encontram-se com sintomas internalizantes importantes, como a depressão, necessitando de intervenção psicológica individualizada, como atendimento psicoterápico, focalizando o manejo adequado de tais sintomas.

Os distúrbios externalizantes também se mostraram significativamente associados às crianças obesas deste estudo. Convém salientar que esse dado é compatível com a literatura, que aponta que problemas comportamentais são encontrados, com maior frequência, entre as crianças obesas, quando avaliadas pelos pais ou professores (Epstein et al., 1996; Stradmeijer et al., 2000). Esses resultados evidenciam a necessidade de implementar estratégias específicas de tratamento, como o atendimento psicológico a essas crianças, 46 enfocando a diminuição de tais problemas, e orien- tações específicas aos pais, com o objetivo de ensinar estratégias adequadas para lidarem com os filhos.

Assim, os distúrbios comportamentais totais, neste estudo, foram significativamente associados à obesidade infantil, sendo que eles correspondem a uma visão geral dos comportamentos desviantes da criança e de seu funcionamento global (Marinho, 1999). É importante que se diga que o mesmo instrumento utilizado nesta pesquisa fez parte de um estudo realizado por Stradmeijer et al. (2000). Esses autores verificaram também que, quando avaliadas por seus pais e professores, as crianças obesas apresentavam mais problemas comportamentais do que as crianças de peso adequado.

Os resultados apontaram a necessidade de o psicólogo se inserir em programas que trabalhem com medidas preventivas, visto que a obesidade está fortemente associada a problemas emocionais, ao sofrimento e à estigmatização. As medidas preventivas são amplas e podem incluir campanhas que abordem estigmatização, problemas psicossociais, mudanças no estilo de vida e orientações específicas para pais, professores, profissionais da saúde e comunidade.

Outra vertente de atuação do psicólogo em relação à obesidade infantil é o atendimento em grupo com equipes multidisciplinares, com enfoque no manejo da obesidade e de suas consequências tanto físicas quanto psicológicas; mais especificamente, o grupo permite a troca de experiências entre os participantes, facilita a adesão ao tratamento e pode contribuir para a melhora da competência social das crianças.

\section{Considerações Finais}

Este estudo foi elaborado com grupo-controle, sendo que os dois grupos avaliados apresentaram características semelhantes em relação aos aspectos demográficos, o que trouxe maior consistência aos dados.

Evidenciou-se maior número de crianças obesas com sintomas de depressão. O mesmo ocorreu em relação aos déficits de competência social e aos distúrbios comportamentais, tanto internalizantes quanto externalizantes.

Tais dados foram confirmados na análise multivariada, que indicou que as variáveis depressão e distúrbios 
comportamentais internalizantes foram as preditoras da ocorrência da obesidade infantil na amostra estudada.

Pode-se concluir que, neste estudo, houve diferença significativa entre crianças obesas e não obesas em relação às variáveis psicológicas estudadas. Assim, preconiza-se a importância da elaboração de programas preventivos nesta área, tanto para os aspectos da obesidade quanto para os problemas psicológicos, e de incluir nos atendimentos a essas crianças protocolos que avaliem, corretamente, aspectos psicológicos infantis, permitindo, assim, tratamento efetivo e adequado.

Considerando-se que pesquisas como esta sejam relevantes para elucidar aspectos relativos ao funcionamento psicológico e social de crianças obesas, e tendo em vista o aumento da prevalência da obesidade infantil e seu difícil manejo, acredita-se que a realização de futuras pesquisas, nesta área, possa complementar os resultados obtidos.

\section{Referências}

Abrantes, M. M., Lamounier, J. A., \& Colosimo, E. A. (2002). Prevalência de sobrepeso e obesidade em crianças e adolescentes das regiões sudeste e nordeste. Jornal de Pediatria, 78 (4), 335-340.

Anton, S. D., Newton, R. L. Jr., Sothern, M., Martin, C. K., Stewart, T. M., \& Williamson, D. A. (2006). Association of depression with body mass index, sedentary behavior, and maladaptive eating attitudes and behaviors in 11 to 13-year old children. Eating Weight Disord, 11 (3), e 102-e108.

Bordin, I. A. S., Mari, J. J., \& Caiero, M. F. (1995). Validação da versão brasileira do "Child Behavior Checklist" (CBCL) (Inventário de Comportamentos da Infância e Adolescência): dados preliminares. Revista ABP-APAL, 17 (2), 55-66.

Brownell, K. D., \& O'Neil, P. M. (1999). Obesidade. In D.H. Barlow. Manual clínico dos transtornos psicológicos (2a. ed., pp.355-403). Porto Alegre: Artmed.

Candy, C. M., \& Fee, V. E. (1998). Underlying dimensions and psychometric properties of the eating behaviors and body image test for preadolescent girls. Journal ofClinical Child Psychology, 27 (1), 117-127.

Centers for Disease Control and Prevention. (2007). Department of Health and Human Services USA. About $B M I$ for children and teens. Atlanta: Centers for Disease Control and Prevention, 2000. Retrieved February 18,
2008, from http://www.cdc.gov/nccdphp/dnpa/bmi/ childrens_BMI/about_childrens_BMI.htm

Csabi, G., Tenyi, T., \& Molnar, D. (2000). Depressive symptoms among obese children. Eating Weight Disorders, 5 (1), 43-45.

Damiani, D. (2002). Obesidade: fatores genéticos ou ambientais? Pediatria Moderna, 38 (3), 57-80.

Epstein, L. H., Myers, M. D., \& Anderson, K. (1996). The association of maternal psychopathology and family socioeconomic status with psychological problems in obese children. Obesity Research, 4 (1), 65-74.

Erermis, S., Cetin N., Tamar, M., Bukusoglu, N., Akdeniz, F., \& Goksen D. (2004). Is obesity a risk factor for psychopathology among adolescents? Pediatrics International, 46 (3), 296-301.

Erickson, S. J., Robinson, T. N., Haydel, K. F., \& Killen, J. D. (2000). Are overweight children unhappy. Archives of Pediatrics and Adolescent Medicine, 154 (9), 931-935.

Falcone, E. M. O. (2000). A evolução das habilidades sociais e o comportamento empático. In E. F. M. Silvares (Org.), Estudos de caso em psicologia clínica comportamental infantil (pp.49-77). Campinas: Papirus.

Ferriani, M. G. C., Dias, T. S., Silva, K. Z., \& Martins, C. S. (2005), Auto-imagem corporal de adolescentes atendidos em um programa multidisciplinar de assistência ao adolescente obeso. Revista Brasileira SaúdeMaterno Infantil, 5 (1), 27-33.

Gouveia, V.V. (1995). Inventário de depressão infantil: estudo de adaptação com escolares de João Pessoa. Jornal Brasileiro de Psiquiatria, 44 (7), 345-349.

Keller, C., \& Steves, K. R. (1996). Assessment, etiology, and intervention in obesity in children. Nurse Practitioner, 21 (9), 31-42

Khaodhiar, L., McCowen, K. C., \& Blackburn, G. L. (1999). Obesity and its comorbid conditions. Clinical Cornerstone, 2 (3), 17-31.

Kovacs, M. (1982). Children depression inventory: manual. New York: MHS.

Lee, F. I., Curatolo, E., \& Freedsich, S. (2000). Transtornos afetivos. Revista Brasileira de Psiquiatria, 22 (Supl2), 524-527.

Luiz, A. M. A. G., Gorayeb, R., Liberatore Júnior, R. D. R., \& Domingos, N. A. M. D. (2005). Depressão, ansiedade e competência social em crianças obesas. Estudos de Psicologia (Natal), 10 (1), 35-39.

Marinho, M. L. (1999). Orientação de pais em grupo: intervenção sobre diferentes queixas comportamentais infantis. Tese de doutorado não-publicada, Universidade de São Paulo.

Mello, E. D., Luft, V. C., \& Meyer, F. (2004). Obesidade infantil: como podemos ser eficazes? Jornal de Pediatria (Rio de Janeiro), 80 (3), 173-82.

Miyazaki, M. C. O. S. (1993). Enfoque psicossocial da doença crônica: um estudo sobre depressão em pacientes pediátricos asmáticos e suas mães. Dissertação de mestrado não-publicada, Pontifícia Universidade Católica de Campinas. 
Mustillo, S., Worthman, C., Erkanli, A., Keeler, G., Angold, A., \& Costello, E. J. (2003). Obesity and psychiatric disorder: developmental trajectories. Pediatrics, 111 (4 Part1), 851-859.

Neutzling, M. B., Taddei, J. A., Rodrigues, E. M., \& Sigulem, D. M. (2000). Owerweight and obesity in Brazilian adolescents. International Journal of Obesity and Related Metabolic Disorders, 24 (7), 869-874.

Rose, R. M. (1988). Psicoendocrinologia. In J. D. Wilson \& D. W. Foster. Tratado de endocrinologia (pp.809-841). São Paulo: Manole.

Stradmeijer, M., Bosch, J., Koops, W., \& Seidell, J. (2000). Family functioning and psychosocial adjustment in overweight youngsters. The International Journal of Eating Disorders, 27 (1), 110-114.
Strauss, R. (1999). Childhood obesity. Current Problems in Pediatrics, 29 (5), 5-29.

Suné, F. R., Dias-da-Costa, J. S., Olinto, M. T. A., \& Pattussi, M. P. (2007). Prevalência e fatores associados para sobrepeso e obesidade em escolares de uma cidade do Sul do Brasil. Cadernos de Saúde Pública, 23 (6), 1361-1371.

van Vlierberghe, L., Braet, C., \& Mels, S. (2008). Psychiatric disorders and symptom severity in referred versus non-referred overweight children and adolescents. European Child Adolescents Psychiatry, 18 (3), 164-173.

Recebido em: 3/4/2008

Versão final reapresentada em: 26/2/2009

Aprovado em: 5/3/2009 